Leserbrief

Z Gerontol Geriat 2019 · 52:200

https://doi.org/10.1007/s00391-019-01514-6

Online publiziert: 6. Februar 2019

(c) Springer Medizin Verlag GmbH, ein Teil von Springer Nature 2019

F. Baierlein - J. Masuch - M. Gosch · K. Singler

Universitätsklinik für Geriatrie, Paracelsus Medizinische Privatuniversität Nürnberg, Klinikum Nürnberg, Nürnberg, Deutschland

\title{
Kunsttherapie in der nichtmedikamentösen Therapie chronischer Schmerzen
}

\section{Leserbrief zu}

Drebenstedt C (2018) Nichtmedikamentöse Schmerztherapie bei chronischem Schmerz. Z Gerontol Geriat 51: 859-864. https://doi. org/10.1007/s00391-018-01465-4

Mit großem Interesse haben wir Ihren Artikel zur nichtmedikamentösen Therapie bei chronischen Schmerzen gelesen. Diese haben, wie von Ihnen dargestellt, einen großen Stellenwert, wenngleich die wissenschaftliche Evidenz eher schwach ist.

Da in Ihrem Artikel sehr umfassend auf zur Verfügung stehende therapeutische Möglichkeiten eingegangen wurde, darf die Kunsttherapie nicht vergessen werden. Im Rahmen der Kunsttherapie werden ebenfalls Wirkmechanismen auf das körperliche und emotionale Wohlbefinden verschiedener Patientengruppen beobachtet. So beschreiben Plecity et al. [1] in einer Pilotstudie, dass Kunsttherapie als Teil eines multimodalen Behandlungskonzeptes in der Psychosomatik zur Verbesserung der Stimmung und körperlichen Symptomatik beitragen konnte. „Die Entwicklung und Validierung eines Fragebogens zur Effekt- und Verlaufsmessung von Kunsttherapie" durch Schwerdt et al. [2] führte weiterhin zu der Erkenntnis, dass Kunsttherapie das Schmerzerleben von chronischen Schmerzpatienten durch den kreativen Prozess beeinflussen kann. Die Autoren gehen hierbei von verschiedenen „Handlungs- und Erlebensprozessen“ aus, die durch die Kunsttherapie entsprechend modifiziert werden.
Im Rahmen einer Studei an der geriatrischen Tagesklinik am Klinikum Nürnberg kommt die Kunsttherapie bei chronischen Schmerzpatienten seit nunmehr 15 Monaten zum Einsatz.

Vonseiten der Patienten wird die Kunsttherapie sehr gut angenommen, und erste Zwischenergebnisse weisen auch auf eine Verbesserung psychischer Assessmentparameter hin.

\section{Korrespondenzadresse}

\section{PD Dr. med. K. Singler}

Universitätsklinik für Geriatrie, Paracelsus Medizinische Privatuniversität Nürnberg, Klinikum Nürnberg

Prof. Ernst Nathan Straße 1, 90419 Nürnberg, Deutschland

Katrin.Singler@klinikum-nuernberg.de

Interessenkonflikt. F. Baierlein, J. Masuch, M. Gosch und K. Singler geben an, dass kein Interessenkonflikt besteht.

\section{Literatur}

1. Plecity DM, Danner-Weinberger A, Szkura L, von Wietersheim J (2009) Die Auswirkungen der Kunsttherapie auf das körperliche und emotionale Befinden der Patienten - eine quantitative und qualitative Analyse. Psychother Psych Med 59:364-369. https://doi.org/10.1055/s-20081067573

2. Schwerdt C, Wirtz M, Grabbe Y, Gralow I (2002) Kunsttherapie in der Behandlung chronifizierter Schmerzpatienten. Entwicklung und Validierung eines Fragebogens zur Kunsttherapie. Psychotherapeut 47:301-309. https://doi.org/10.1007/ s00278-002-0241-x 